\title{
A Preterm Infant with Secondary Carnitine Deficiency due to MCT Formula - Effective Treatment of L-Garnitine -
}

\author{
Akira Ishida, Atsuko Goto, Yasushi Takahashi, Wako \\ Nakajima, Hirokazu Arai, Yusaku Tazawa and Goro \\ TAKADA \\ Department of Pediatrics, Akita University School of \\ Medicine, Akita 010
}

\begin{abstract}
Ishida, A., Goto, A., Takahashi, Y., Nakajima, W., Arai, H., Tazawa, Y. and Takada, G. A Preterm Infant with Secondary Carnitine Deficiency due to MCT Formula - Effective Treatment of L-Carnitine - . Tohoku J. Exp. Med., 1994, 172 (1), 59-64 — We report a preterm infant who was prescribed an MCT formula and subsequently developed carnitine deficiency with liver dysfunction and an elevation of serum CK level. A male infant who had been born at 24 weeks' gestation with birth weight $799 \mathrm{~g}$, was fed with an MCT formula containing $76.8 \%$ of all kinds of lipids, because of his steatorrhea after the 30th day. On the 100th day, he was noted hepatomegaly and elevation of serum levels of AST, ALT and CK. The needle biopsy of the liver indicated the existence of the liver damage. He showed low serum carnitine with high urinary loss of acylcarnitine and dicarboxylic aciduria. Administration of L-carnitine was an effective treatment. The carnitine deficiency might be exaggerated by an increased urinary loss of acylcarnitine. We should be cautious of the risk of carnitine deficiency in preterm infants during prolonged use of MCT formula. —— preterm infant; carnitine deficiency; L-carnitine; MCT
\end{abstract}

The use of medium-chain triglyceride (MCT) solution or oil as a fuel source in preterm infants is widely accepted (Borum 1992). Recent evidence suggests that carnitine plays an important role in the metabolism of medium-chain fatty acids as well as long-chain fatty acids (Rebouche et al. 1990). Thus, any MCT formula would increase the urinary excretion of acylcarnitine (Rebouche et al. 1990). Urinary loss of carnitine may induce clinical carnitine deficiency in preterm infants, who are known to be in low carnitine state (Penn et al. 1985). We report a preterm infant who developed severe symptoms of carnitine deficiency probably because of prolonged feeding with an MCT formula for fat malabsorption.

Received September 28, 1993; revision accepted for publication December 20, 1993. Address for reprints: Akira Ishida, MD, Department of Pediatrics, Akita University School of Medicine, 1-1-1 Hondo, Akita 010, Japan. 


\section{Case Report}

A male infant was born at 24 weeks' gestation to a gravida 3, para 2 healthy mother. Delivery was by emergency cesarean section because of a forelying umbilical cord. Apgar scores were 1 at $1 \mathrm{~min}$. and 7 at $5 \mathrm{~min}$. Birth measurements were as follows: weight 799 $\mathrm{g}$, length $32.0 \mathrm{~cm}$ and head circumference $23.2 \mathrm{~cm}$. Mechanical ventilation was performed for 76 days due to apnea of prematurity and chronic lung disease. Breast-milk feeding began through an oral gastric tube on the 8th day and the infant was fed a preterm-formula (Formula A: GPT milk ${ }^{\circledR}$, Morinaga Milk Industry Company, Tokyo, see Table 1) after the 14th day of life.

On the 30th day, the infant began to defecate frequent, bulky, and greasy stools. These stools contained readily visible droplets of fat, which were stained positively by Sudan III. The preterm formula was changed to a formula for fat malabsorption (Formula B: L-MCT milk ${ }^{\circledR}$, Wakohdo Inc., Tokyo: cow's milk-derived formula containing $17.3 \mathrm{~g}$ of MCT per $100 \mathrm{~g}$ of powder, which contains $140 \mathrm{nmol} / \mathrm{ml}$ of carnitine (Sugiyama et al. 1984)). Steatorrhea was improved to normal yellow stool several days after the change in formula.

On the 100th day, his liver was palpable $3 \mathrm{~cm}$ below the right costal margin. AST, ALT and total bilirubin were $248 \mathrm{U} / 1,85 \mathrm{U} / 1$ and $5.7 \mathrm{mg} / 100 \mathrm{ml}$, respectively. Serum creatine phosphokinase (CK) increased over 1,500 U/l. He showed no sign of hypotonia nor cardiac failure.

On the 147th day, needle biopsy of the liver showed an intrahepatic accumulation of bile, persistent extramedullary hematopoiesis and mild periportal fibrosis. Urinary organic acid analysis by gas chromatography with mass-spectrometry revealed an increased excre-

TABLE 1. Contents of three formulas $(\mathrm{g} / 100 \mathrm{~g}$ powder)

\begin{tabular}{lccc}
\hline & Formula A & Formula B & Formula C \\
\hline Protein & 15 & 13 & 14 \\
Fat & 23 & 22 & 23 \\
MCT content & 4 & 17 & 11 \\
Linoleic acid & 3 & 3 & 3 \\
Carnitine $^{\mathrm{a}}$ & 23 & 15 & 19 \\
Calory intake $^{\mathrm{b}}$ & 492 & 493 & 493 \\
\hline
\end{tabular}

${ }^{\mathrm{a}} \mathrm{mg} / 100 \mathrm{~g}$ powder

${ }^{\mathrm{b}} \mathrm{kcal} / 100 \mathrm{~g}$ powder

TABLE 2. Serum and urinary concentration of carnitine

\begin{tabular}{lrrrrrr}
\hline & \multicolumn{2}{c}{ Formula B } & \multicolumn{2}{c}{ Formula C } & \multicolumn{2}{c}{$\begin{array}{c}\text { Formula C+ } \\
\text { L-Carnitine }\end{array}$} \\
\cline { 2 - 8 } & Free & Acyl & Free & Acyl & Free & Acyl \\
\hline Serum $(\mathrm{nmol} / \mathrm{ml})$ & 13.8 & 6.3 & 18.1 & 6.5 & 110.1 & 46.6 \\
Urine $(\mathrm{nmol} / \mathrm{mg}$ creatinine) & 71.4 & 687.5 & 126.3 & 440.9 & 1277 & 1560 \\
\hline
\end{tabular}

age matched normal value $(n=42)$ : serum free carnitine $30.7 \pm 8.6$, acyl carnitine

$5.0 \pm 4.9(\mathrm{nmol} / \mathrm{ml})$

urine free carnitine $67.5 \pm 40.8$, acyl carnitine $13.8 \pm 18.7$ (nmol/mg creatinine) 


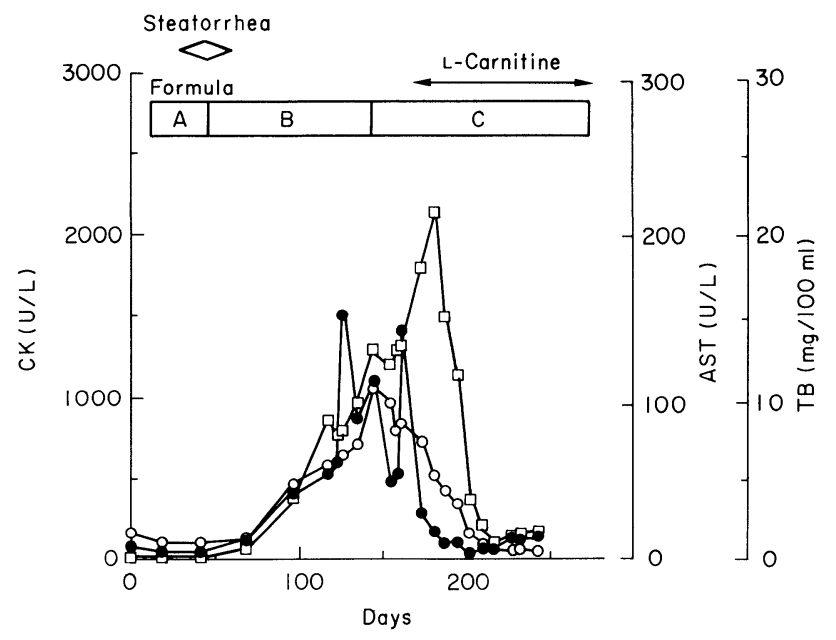

Fig. 1. The clinical course and changes in serum levels of aspartate aminotransferase (AST, $\square-\square)$, total bilirubin (TB, O-O) and creatine phosphokinase (CK, - - ). A, B and C indicate Formula A, B and C, respectively. We began to give him a Formula B after steatorrhea. After that, AST, TB and CK increased gradually. Formula $\mathrm{C}$ could not improve these data, but abministration of L-carnitine decreased the elevations of AST, TB and CK.

tion of dicarboxylic acids (adipic, suberic and sebacic acids) in his urine. Adipic and suberic acids were eleven to seventeen times greater than normal. Sebacic acid is not excreted in normal urine. As shown in Table 2, serum levels of free carnitine were very low and urinary excretions of acylcarnitine were very high in the periods without exogenous carnitine administration.

On the 150th day, Formula C (mixture of Formula A with equal volume of Formula B) was began, but neither an improvement of liver function nor a decrease of serum CK level was observed.

From the 160 th day, L-carnitine $(80 \mathrm{mg} / \mathrm{kg} /$ day) was added to his formula, as shown in Fig. 1. By the 200th day, liver function improved and serum CK fell into normal range. Serum levels of free and acylcarnitine increased at ten times before L-carnitine administration, and the urinary excretion also increased.

Serum and urinary carnitine were assayed by a minor modification of the method described by Parvin and Pande (1977).

\section{Discussion}

Preterm infants, especially extremely low birth-weight infants, are more likely to be suffered from various metabolic abnormalities (Goto et al. 1992). In fat metabolism, carnitine plays a very important role as a carrier of long chain fatty acids into mitochondria. Carnitine state is known to reflect its supply, particularly in premature infants, who have originally very low carnitine tissue concentrations (Penn et al. 1985). So that, if premature infants are low in carnitine intake or high in urinary loss, they will easily fall into a carnitine deficient state (Penn et al. 1981, 1985). Urinary excretions of dicarboxylic acids 
and acylcarnitine with low serum free carnitine in our case indicate carnitine insufficiency. In normal term infants, a carnitine deficient state, do not necessarily associate with clinically recognizable abnormalities in fat metabolism. However, in high risk populations such as preterm infants or infants who are genetically or environmentally stressed (infection, injury, prolonged fasting, parenteral alimentation, or malnutrition), abnormalities may be more severe, and may produce a clinically relevant carnitine deficiency (Olson et al. 1989). Carnitine deficiency induces an elevation of serum CK originating from muscle and liver dysfunction (Karpati et al. 1975; Engel 1986). Matsuo et al. reported an extremely low birth-weight infant who developed cholestasis and was successfully treated with D,L-carnitine (Matsuo et al. 1989). In our case, elevated serum levels of CK and of AST and ALT suggest muscle destruction and liver dysfunction, which was proved by the needle liver-biopsy, showing an intrahepatic accumulation of bile, persistent extramedullary hematopoiesis and mild periportal fibrosis.

It was previously reported that oral medium-chain triglyceride feeding could provoke dicarboxylic aciduria in preterm neonates (Whyte et al. 1986; Böhles et al. 1987). Concentrations of acylcarnitine were greatly increased above normal with free carnitine concentrations ranging from undetectable to supranormal values in urine of infants with increased dietary intake of MCT (Rebouche et al. 1990). Increased urinary excretion of dicarboxylic acids and acylcarnitine indicates an imbalance of mitochondrial mass action homeostasis (Chalmers et al. 1984). Dietary intake of MCT increases acyl moieties in mitochondria with marked reduction of free CoA (Rössle et al. 1990). Carnitine removes these excess acyl groups as acylcarnitine (Rebouche et al. 1990). The increase in the acyl/free carnitine ratio that is related to the accumulation of acyl-and acetyl-CoA in the mitochondria (Rössle et al. 1990), also indicates the dysfunction of mitochondrial fatty acid oxidation. Carnitine acts as a sink for short-chain acyl moieties generated by $\beta$-oxidation, thus relieving intramitochondrial coenzyme $\mathrm{A}$ for participation in fatty acid activation, i.e., $\beta$-oxidation, and the Krebs cycle (Rebouche et al. 1990). As availability of free coenzyme A diminishes, the efficiency of mitochondrial utilization of medium-chain fatty acids decreases, thus increasing the extramitochondrial pool of medium-chain fatty acids and the rate of $\omega$-oxidation (Rebouche et al. 1990). Dicarboxylic acids, such as adipic-, suberic- and sebacic acids are excreted in urine during microsomal $\omega$-oxidation, a salvage pathway, in case of a limited capacity or interruption of $\beta$-oxidation (Böhles et al. 1987). Therefore, MCT formula may play a role inducing a carnitine deficient state.

Oral L-carnitine supplement to our patient resulted in improvement in clinical and laboratory findings, such as liver function tests and serum levels of CK and free carnitine, while augmenting the urinary excretion of acylcarnitine. Carnitine supplementation led to a further increase in the dicarboxylic acids in 
rats receiving MCT (Böhles et al. 1987). As shown by Chalmers et al., the fact that an additional oral load of exogenous L-carnitine resulted in an even greater excretion of acylcarnitine would further support for the concept of carnitine insufficiency in our patient (Chalmers et al. 1984). The high carnitine concentration in the serum in spite of low tissue carnitine of preterm infants reflects an immaturity of the mechanisms of carnitine uptake and of storage in the tissues (Rubaltelli et al. 1987). Therefore, any premature babies may need to be maintained a higher level of serum carnitine when their renal carnitine leak increases.

It could be dangerous to use a large amount of MCT formula for some preterm infants without sufficient carnitine supplementation.

\section{References}

1) Böhles, H., Akcetin, Z. \& Lehnert, W. (1987) The influence of i.v. MCT and carnitine on the excretion of dicarboxylic acids. J. Parenter. Nutr., 11, 46-48.

2) Borum, P.R. (1992) Medium-chain triglycerides in formula for preterm neonates: Implications for hepatic and extrahepatic metabolism. J. Pediatr., 120, S139-145.

3) Chalmers, R.A., Roe, C.R., Stacey, T.E. \& Hoppel, C.L. (1984) Urinary excretion of l-carnitine and acylcarnitines by patients with disorders of organic acid metabolism: Evidence for secondary insufficiency of l-carnitine. Pediatr. Res., 18, 1325-1328.

4) Engel, A.G. (1986) Carnitine deficiency syndromes and lipid storage myopathies. In: Myology, edited by A.G. Engel \& B.Q. Banker, Vol. 2, McGrow Hill Book Company, New York, pp. 1663-1696.

5) Goto, A., Ishida, A., Goto, R., Hayasaka, K., Nanao, K., Yamashita, A., Yamaguchi, S. \& Takada,G. (1992) Transient 5-oxoprolinuria in a very low-birthweight infant. J. Inher. Metab. Dis., 15, 284-285.

6) Karpati, G., Carpenter, S., Engel, A., Watters, G., Allen, J., Rothman, S., Klassen, G. \& Mamer, O.A. (1975) The syndrome of systemic carnitine deficiency. Neurology, $25,16-24$.

7) Matsuo, M., Saiki, K., Momota, T., Ishida, A., Kanazawa, K., Murakami, R., Nakamura, H. \& Matsuo, T. (1989) Transient carnitine-responsive medium-chain dicarboxylic aciduria in an infant with cholestasis, hypoglycemia and cardiac failure. Acta Pediatr. Jpn., 31, 211-215.

8) Olson, A.L., Nelson, S.E. \& Rebouche, C.J. (1989) Low carnitine intake and altered lipid metabolism in infants. Am. J. Clin. Nutr., 49, 624-628.

9) Parvin, R. \& Pande, S.V. (1977) Microdetermination of (-) carnitine and carnitine acetyltransferase activity. Anal. Biochem., 79, 191-201.

10) Penn, D., Schumidt-Sommerfeld, E. \& Pascu, F. (1981) Decreased tissue carnitine Concentration in newborn infants receiving total parenteral nutrition. J. Pediatr., 98, 976-981.

11) Penn, D., Ludwigs, B., Schumidt-Sommerfeld, E. \& Pascu, F. (1985) Effect of nutrition on tissue carnitine concentrations in infants of different gestational ages. Biol. Neonate, 47, 130-135.

12) Rebouche, C.J., Panagides, D.D. \& Nelson, S.E. (1990) Role of carnitine in utilization of dietary medium-chain triglycerides by term infants. Am. J. Clin. Nutr., 52, 820-844.

13) Rössle, C., Carpentier, Y.A., Richelle, M., Dahlan, W., D’Attellis, N.P., Fürst, P. \& Elwyn, D.H. (1990) Medium-chain triglycerides induce alterations in carnitine metaboliosm. Am. J. Physiol., 258, E944-E947. 
14) Rubaltelli, F.F., Orzali, A., Rinaldo, P., Donzelli, F. \& Carnielli, V. (1987) Carnitine and the premature. Biol. Neonate, 52, Suppl. 1, 65-77.

15) Sugiyama, N., Suzuki, K. \& Wada, Y. (1984) Carnitine concentration of prepared formula. J. Jpn. Ped. Soc., 88, 1968-1971. (in Japanese)

16) Whyte, R.K., Whelan, D., Hill, R. \& McClorry, S. (1986) Excretion of dicarboxylic and $\omega-1$ hydroxy fatty acids by low birth weight infants fed with medium-chain triglycerides. Pediatr. Res., 20, 122-125. 